\title{
On the role of awareness systems for supporting parent involvement in young children's schooling
}

\author{
Vassilis-Javed Khan, Panos Markopoulos and Berry Eggen \\ Eindhoven University of Technology, Eindhoven, The Netherlands
}

\begin{abstract}
This paper reports on an investigation of how Awareness Systems can support communication between busy parents and young children at school. The study involved the deployment and test of a rudimentary awareness system so that surveying opinions and wishes regarding this type of technology would be grounded upon concrete experiences. The prototype was installed in a classroom for two weeks and connected five families with their children. Overall, parents appreciated receiving awareness information and did not experience it as an undesirable distraction. Both they and their children did not experience privacy problems. They raised concerns about the possibility of children becoming more dependent upon their parents from such technology and stated they would not want to receive information that they would not be able to react upon.
\end{abstract}

\section{Introduction}

In recent years there has been an increasing interest in technologies that support sustained communication channels between individuals or groups that allow them to build up and maintain a mental model of the activities of each other. Often these technologies are discussed under the label of "awareness systems", in juxtaposition to solutions that support the efficient and goal directed exchange of information. Early research on Media Spaces examined the use of long-term video and audiolinks supporting co-workers, [1] or in a domestic context [2], [3].

Awareness systems are usually conceived as requiring low effort: information is usually captured semi-autonomously and the display of it is usually designed to be non-disruptive. Often the terms 'peripheral display' and 'peripheral awareness' are used respectively to suggest that the information they present furnishes the periphery of someone's attention and is easily brought to the foreground when needed or that the person viewing it has to expend minimal effort and almost without noticing build up awareness of another person or group.

llease we the following format when cing this chapler:

Khan, V.-J., Markopoulos, P., Eggen, B., 2007, in IFIP International Federation for Information Processing, Volume 241, Home Informatics and Telematics: ICT for the Next Billion, eds. Venkatesh, A., Gonsalves, T., Monk, A., Buckner, K., (Boston: Springer), pp. 91-101. 
The interest in awareness systems can be seen as symptomatic of an ever increasing availability of information and communication technology, in different contexts, for different user groups. The present research investigates the potential of awareness systems to support busy working families with children. We are especially interested in how to provide technological solutions that will stimulate and facilitate parental involvement in children's lives. Increased parental involvement is recognized by social scientists to provide benefits for the development of children socially and academically [4], [5].

There have been several attempts to support communication between parents and children and to inform parents of school activities. For example, [6] report the use of voice-mail to support communication between teachers and parents. A voice-mailmessaging application enabled parents and teachers exchange messages asynchronously. The experimental use of the system was reported to result in a steep increase of information exchange between them. School failure of students dropped and there was an increase in the number of students who became eligible for academic honor. This case suggests the potential benefits of the deployment of modern communication technologies.

While such a direct system such as a voice-mail has a lot of potential it only supports communication between teacher and parent and it requires an explicit effort by the teacher. This kind of effort may be hard to sustain over longer periods of time and to scale up for a large number of pupils. Awareness systems connecting parents to their children through the day could address this limitation, by supporting the semi-automatic capture of relevant information and its use as a complement to extant communication channels, including face-to-face communication or even voice-mail.

The auxiliary nature of awareness information has to be stressed here. Parents and children who are not separated by distance or other social problems (like a divorce) are most likely to get ample opportunity to communicate with each other. However, especially for younger children, parents may find it problematic to find out what is happening through the day, what are the problems their children are facing, what are their successes and joys. It is well known and confirmed by recent studies, e.g., Hoenderdos et al. [7], how difficult it is for parents to obtain answers to the question "how was your day?" beyond one-word descriptions such as 'fine', 'ok'. Hounderdos et al. proposed a sound capture device that children could use through the day to record sounds from their environment. The main concept (that was never realized and tested) was to play these sounds for parents later in the day, letting them provide triggers for their conversations. Another interesting project [8] examined the exchange of video clips between children and other family members to support them in feeling connected.

The main difference of our motivation with the aforementioned projects lies in the explicit/intentional nature of communication. We are interested in supporting automatic exchange of awareness information rather than have explicit communication. The advantages are obvious for the group of people we are focusing: 
saving time and effort for parents and teachers while keeping informal communication channels open. However, challenges lie in what content should such systems exchange, how it will be used and what type of privacy concerns arise.

The present study builds on an earlier interview study regarding the communication needs of busy parents [9]. In that study semi-structured interviews were held with 20 parents and were analyzed qualitatively. The analysis inventorized communication needs that are not met sufficiently by current technology and explored the potential and drawbacks of awareness systems for this target user group. One of the main findings was that parents wish for more awareness of what happens at school. There were several questions left open; specifically some skepticism towards the potential role of awareness systems was expressed.

More specifically, parents were skeptical of an 'always-on' communication channel. For example, interviewees suggested that when at work they might prefer not to have information they cannot react to (e.g., if their child is missing them) or that the constant availability of information about their children might distract them from their work, disturbing the boundaries they try to maintain between their work and their private lives. Parental involvement in children's lives emerged as a category of particular importance, influencing the behavior, communication and awareness needs of the working parent throughout the day. Finally, the exchange of experiences especially during dinner, was found to be a valued ritual among our participants. The present study aimed to probe deeper in those issues and examine when awareness information is distracting or undesirable and what kind of information may be most valued.

While very informative, these interviews relied on self-reported beliefs and attitudes expressed outside a specific context and without reference to a specific technology. Interviewed parents found it hard to relate to the concept of awareness systems and as a result the related discussion was hypothetical and results not very reliable. We felt that exposing parents to using a system comparable to the class of systems we envision would provide richer and more reliable outcomes. In the remainder of this paper, we describe how we set up a simple awareness service for parents and their children and the reactions we obtained from a two week field trial. We do not suppose that this specific system is the solution they require yet it embodies some important characteristics of the type of systems we are interested in and as such serves to solicit relevant reactions and opinions from them.

\section{Method}

The study aimed to explore how busy parents and their children would experience a system providing awareness information about the children during school hours. A 
simple awareness system was built and parents were encouraged to use it for two weeks, after which they were interviewed regarding their experience. The study aimed to answer the following questions:

- Is there a need of parents having awareness information during the day about their children?

- Are such systems disruptive for the parents?

- Are such systems perceived privacy-threatening for children?

Accepting that the awareness information we provided was very basic and limited to what was easy to build in a short time, we set out to explore what kind of awareness information would be more valuable for our informants than what we could provide at this stage of our research.

\subsection{Participants}

We recruited five families from an international school. Our target group was "busy parents". More specifically we were looking for participants that:

- Are married or cohabiting,

- Have at least one dependent child,

- Both parents in the household work a minimum of 20 hours a week,

- Have children between the ages of 6 and 10 .

We covered all of our requirements except the third one. Three of the couples we recruited had only one member who was working full time whereas the other was not working. We had in total five children and eight parents (three couples and two parents). The children were ten years old studying at the 7 th class. They were fluent in English. It was a culturally mixed group consisting of one Korean, one Taiwanese, one American and two British. The average age of the participating parents was 43; they have been married on average 13,8 years and have on average of 2,2 children. Our participants were highly educated and hold higher than average positions in their employment.

\subsection{Process}

First, we held a briefing session at school. We handed the prototype PC application in a CD the week before the study was executed. One of the parents for each child involved in the study participated in the briefing session. During the session we presented background information to the research, introduced the study and answered questions they had.

By handing out the $\mathrm{CD}$ a week, we gave them the opportunity to install it and check whether everything was running properly prior to the actual study. Two participants had some technical problems but were promptly solved. One participant had several computers and decided not to install it at all, without informing us. We found this out only at the end. Another participant installed it but the connection was 
probably blocked by the firewall of his company. All this happened despite that we contacted the participants during the installation and at the first week through email about problems they might be experiencing.

We let parents experience the prototype for one week. At the end of the first week parents were asked to complete a web questionnaire. At the end of the second week we interviewed the parents. We analyzed those interviews qualitatively. As part of the interview we presented to parents four storyboards illustrating alternative concepts for an awareness system situated in a classroom. We asked the parents to rate each storyboard in scales of the $\mathrm{ABC}$ questionnaire [10] and then had a discussion about the system presented in the storyboard. The storyboards and their results cannot be described in the space of this article.

\subsection{Materials}

\subsubsection{Prototype description}

The prototype we used had three main components. First, was a small Bluetooth headset device. Participating children were asked to turn this on and keep it in their pocket every morning. They carried this device till the end of the school day and then turned it off and left it in the class so that they could turn it on and carry it again next morning.

Next, we installed a PC with an Internet connection and a USB Bluetooth dongle, at the classroom, running XP SP2 and our software which queried every minute for the presence of the children's devices.

After querying, our prototype made a record in a database server at the University $^{2}$. Along with the almost-real-time presence of children the database contained information regarding their day schedule.

The parents' client $^{3}$ was querying through http the database server at the University and was presenting the information to the parents' desktop. We also developed an alternative solution for the parents' in case they could not install our prototype. This was a dynamic web page presenting exactly the same information. We preferred parents to install our prototype in their desktop so that they would not associate this awareness service with a website or another web service. It actually turned out that one parent who had a Macintosh and could not install the software. This parent used the alternative web application.

1 The prototype application running at the $\mathrm{PC}$ in the classroom was developed with $\mathrm{C \#}$. For querying the Bluetooth devices we used OpenNETCF. It was compiled for Windows XP.

${ }^{2}$ MySQL was used as the database server.

${ }^{3}$ The parent's program was developed with C\# and compiled for Windows XP. 
In our prototype, parents could view three possible images. These are shown in Figure 1. By moving the mouse over the image parents would see more detailed information about when was the last check by the PC in the classroom performed. It was developed to be always on top of other windows. Using two buttons parents could minimize or close it.

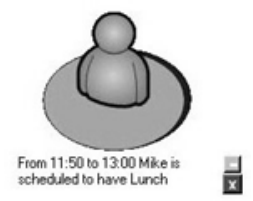

Child's device is detected

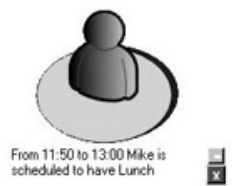

Child's device is not detected

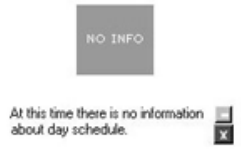

There is a technical problem

Fig. 1. Graphical presentation of awareness information

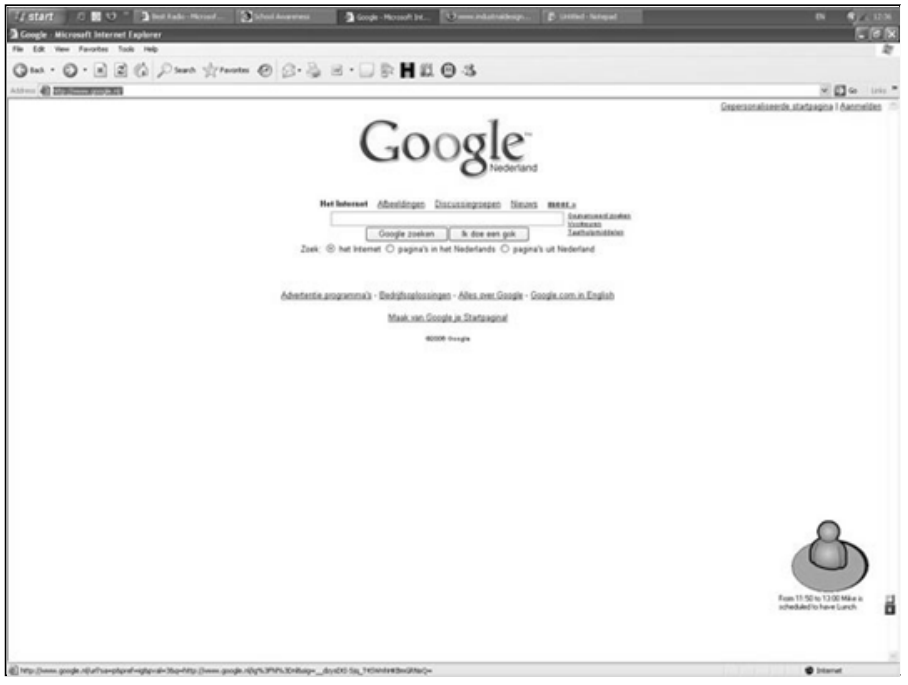

Fig. 2. Screenshot of a parent's desktop

\subsubsection{Web questionnaire}

The web questionnaire included five questions. Participants were asked about their experience till that moment, if they felt the system influenced their conversation with their child, what information was missing and how the child felt about it.

The purpose of having this questionnaire was twofold:

1. Get insight to the participants' experience halfway through the trial.

2. Anchor the final interview to the answers they provided. 


\subsubsection{Interview}

The interview was scheduled after the period of two weeks. It lasted approximately 30 minutes and we began by discussing the answers from the web questionnaire. This was followed by an open discussion about the usage, the feelings and the overall experience of the system followed. Both participating parents and children were present. Children were asked about their experience of the system as well as if they felt their privacy was compromised.

\section{Results}

We must first clarify that we have two groups of users among the participating parents. The ones that used the system in the office (four) and the ones that used it at home (four). The four office users were fathers and the four home users were mothers.

\subsection{On Disruptiveness}

In our earlier interview study mentioned above participants expressed concerns about having a constant information flow. This did not turn out to be an issue for the participants we had. When participants were asked if it was disruptive they mentioned that this was not the case. In the words of a participant: "it was no more demanding than a ping from an email or a PDA or another website, it wasn't sufficiently big".

On the other hand, we must mention that office users complained about the space the image of Figure 1 occupied on their screens. Especially for laptop users; who were compelled to minimize it. Once minimized it was forgotten. The fact that screen space is important for work use denotes the need of having a separate device (e.g., a photo-frame, or a physical output device) for providing awareness information. Participants also mentioned that it did not create a feeling of involvement for them. That might be because when the system was minimized it was forgotten.

Home users reported also that the system was not disruptive. This we believe has to do with the general use of the home computer. It was not constantly used so having such an application running on the computer was a reason for the users to check it. For one participant this system became part of her routine, for the short time of the field study. When going to the kitchen she would also peak at what was going on at the class of her child. 


\subsection{On privacy threat}

No privacy threat was reported by participants. When explicitly asked, both children and parents responded negatively. A child participant mentioned: "it just felt normal". On the other hand, parents thought that if children were older it would have been different. This is consistent with literature on family communication suggesting that children have loose privacy concerns before the age of adolescence [11]. This may explain the different results of [12]; that study involved participants in their adolescence, who experienced serious privacy concerns.

When children were asked about carrying the device and if that created a feeling of being "looked over the shoulder" they unanimously said that this was not the case. Even three of them, in some occasions, forgot that the Bluetooth device was in their pocket and carried it back home. This shows that the device easily fitted their routine and was not something bothering them. In their words: "I didn't even feel it was on me", "I totally forgot about it".

\subsubsection{The feeling of involvement versus the feeling of surveillance}

Though not conclusive, the study was also positive regarding the feelings of involvement in children's lives. In the words of one participant who was a home user: "it actually stirred the spirit of involvement rather than the spirit of surveillance and I didn't expect that". This particular participant liked the fact that the child felt that she was involved in her life.

Moreover this participant reported that the system helped in posing more precise questions to the child about her day. That was the effect of having schedule information. This participant's observation was that the child would easier respond to questions. In the participant's words:

"I always asks them about what happened at school but you sometimes get a word or nothing. If I would ask something like: "what was science like today" it kind of focuses them [referring to the child] cause otherwise the day becomes blur"

Another unexpected observation of the same couple was that they became more sensitized to their child's need to communicate with them. Their child would ask at the evening if they checked the system and asked them for more details about the way it was working.

\subsection{Awareness information that could add value to $A S$ in the context of a school}

Higher precision information would be generally appreciated. Detail was required in the exact location of the children. One of our participants put it very eloquently: "half the story is worse than no story at all". This was a common comment we received by all participants. Conversely, participants expressed a concern; they did not want to have information that would make them worry without the ability of reacting, e.g., if the system would show that there was a scheduled 
outdoor activity whereas the child was sitting in class. Such contradicting information might create a tension. On the one hand it would make the parent feel worried on the other hand the parent knows well that responsibility is handed over to the teacher. We think that this is an important observation that confirms our earlier interview study, reported in [9]. In light of these statements, we believe that an important acceptance factor for awareness systems used in this context is how they impact upon accountability of parents and teachers, and whether they create new concerns and responsibilities for parents who could be expected to react to awareness information shown to them.

An exception to not wanting to have to react to what is displayed to them concerned the safety of the child, e.g., they would want to be informed when the child leaves the school periphery unattended. This was expressed by several participants. One home-participant who was checking the prototype every day would welcome a "red icon" among the grey and green. That red icon would denote danger. Note though that the same participant added that such a system might be a solution for the school rather than the parents.

Another participant stressed the need of having richer information regarding "special occasions". These occasions would include school assemblies, happenings and generally social activities. One more participant wished to be able to observe the social dynamics between the participating children during the day. Another parent mentioned that she would check the prototype when there was a break scheduled because she wanted to be sure her child was out (presumably) playing with other children rather than sitting inside the class. It seems that observing social interactions between the children is a pronounced need for parents. This finding might map to the development phase the children are in.

\section{Conclusions}

Awareness Systems are still in their infancy particularly regarding their use outside collaborative work. The proposed benefits and costs they might bring are still very much hypothesized. Research studies such as the one presented here may help document what needs they can serve and what could be hindrances to their eventual acceptance and adoption.

An outcome of the previous interview study [9] was that busy parents might not want to have continuously available information about their children during their working day. The trial of the prototype suggests that this is not true. Parents valued the awareness information despite the fact that the simplicity of the prototype they experienced prevented it from offering substantial benefits and valuable information to the parents. This seems to be more pronounced for parents using the system from the office. Participants used the prototype more from home rather than the office and were more able to fit its use to their daily routine. However, the fact that participants expressed specific needs for awareness information suggests that such systems can 
bring added value to "busy parents" if appropriately designed and if higher quality information (in accuracy and relevance) would be provided.

The prototype we tested did not raise privacy concerns. A major reason for this was the age of the children concerned. We can expect more privacy concerns for children close to or during adolescence. Hence, more flexible and socially acceptable forms of information capture and communication need to be designed for that group.

Our main conclusion regarding our research aims concerns the need for more specific information from the school such as information about the social development of children.

Currently we are extending our system to be able to provide richer awareness information and to enable the survey of parents' opinions in situ. Such a contextual survey (using diary or experience sampling method) is necessary in order to explore the situatedness of the use of awareness information, that is largely unexplored when surveying the opinions of informants post-hoc. Further we are examining also the requirements of teachers and what their communication and privacy needs are that need to be addressed. Teachers tend to advocate parental involvement and wish to open up communication channels regarding the education of children. On the flip side they also need to protect themselves from excessive communication and excessive workload in providing the required information.

\section{References}

I. S.A. Bly, S.R. Harrison, S. Irwin: Media Spaces: Bringing People Together in a Video, Audio and Computing Environment. Communications of the ACM, 36,1, 1993 p28-47.

2. P. Markopoulos, N. Romero, J. van Baren, W. UJsselsteijn, B. de Ruyter, and B. Farshchian: Keeping in touch with the family: home and away with the ASTRA awareness system. In: Proceedings CHI '04, ACM Press, p. 1351-1354. 2004

3. D. Hindus, S.D. Mainwaring, N. Leduc, A.E. Hagström, and O. Bayley: Casablanca: designing social communication devices for the home. In: Proceedings $\mathrm{CHI}$ '0 I, ACM Press, 2001 p. 325-223.

4. J. Blanchard: The family-school connection and Technology. Paper presented at the Families, Technology, and Education Conference, Washington, DC. 1997

5. K. Hoover-Dempsey, J. M. T. Walker, H. M. Sandler, D. Whetsel, C. L. Green, A. S. Wilkins, and K. E. Closson: Why do parents become involved? Research findings and implications. Elementary School Journal, 106(2); (2005) 105-130

6. J.P. Bauch: Applications of technology to linking schools, families and students. Proceedings of the Families, Technology, and Education Conference. Retrieved Oct. 1,2001 
7. R. Hoenderdos, A. Vermeen, M. Bekker, and A. Pierik: Design for experience: the "Look, mama!" experience, Proceedings of IDC, 2002, p. 4.

8. O. Zuckerman, and P. Maes: CASY: Awareness System for Children in Distributed Families. In Proc. IDC'05, Conference on Interaction Design and Children. Boulder, Colorado, New York, NY, ACM Press. 2005

9. V.J. Khan, P. Markopoulos, S. Mota, W. IJsselsteijn, B. de Ruyter: Intra-family communication needs; how can awareness systems provide support? Proceedings of the 2nd international conference on Intelligent Environments. 2006

10. J. van Baren, W.A. IJsselsteijn, N. Romero, P. Markopoulos, B. de Ruyter: Affective Benefits in Communication: The development and field-testing of a new questionnaire measure. PRESENCE, Aalborg, Denmark, October 2003

11. J.P. Caughlin, and S. Petronio: chapter: "Privacy in Families". In: Vangelisti, A.L., "Handbook of family communication", p. 379. Lawrence Erlbaum Associates. 2004

12. K. Fraser, T. Rodden, and C. O'Malley: Home-school Technologies: considering the Family. IDC'06 